\title{
Precision of and new methods for testing in vitro alloy cytotoxicity
}

\author{
J.C. Wataha ${ }^{1}$, R.G. Craig ${ }^{1}$, C.T. Hanks ${ }^{2}$ \\ ${ }^{1}$ Department of Biologic and Materials Science, ${ }^{2}$ Department of Oral Pathology, Oral Medicine and Oral Surgery \\ University of Michigan, School of Dentistry, Ann Arbor, MI USA
}

\begin{abstract}
Previous studies have utilized in vitro alloy cytotoxicity tests to evaluate dental casting alloys. The purposes of this study were to: (1) evaluate the precision of the optical density and visual tests previously used, (2) evaluate a new test measuring absorbance of solubilized formazan dyes, and (3) test the correlation between these tests for cytotoxicity. Balb/c $3 T 3$ cells were plated in 24-well culture trays at 25,000 cells $/ \mathrm{cm}^{2}$ around ten types of dental casting alloys (six samples/alloy) and incubated for $72 \mathrm{~h}$. Cells were histochemically stained with MTT (3-[4,5dimethylthiazol-2-yl]-2,5-diphenyl tetrazolium bromide)/succinate for $2 \mathrm{~h}$, then fixed, washed, and dried. Toxicity was measured by optical densitometer (OD) scanning, visual assessment, and 560$\mathrm{nm}$ absorbance of DMSO-solubilized dyes. Measurements of rings of inhibition were not used, because they did not provide precise data, and correlated poorly with the other methods. The results were analyzed by ANOVA, Tukey intervals, and coefficients of variation (CV's). MTT required shorter incubation times for adequate staining, allowed for solubilization of the monolayers, and was less expensive than NBT $\left(2,2^{\prime}\right.$-di-p-nitro-phenyl-5,5'diphenyl-3,3'-dimethoxy-[3,3'-dimethoxy-4,4'-biphenylene] ditetrazolium chloride). Results showed that all three methods ranked alloy toxicities similarly $(p=0.05)$. The solubilization method was most discriminating due to lower CV's. Correlation between densitometer and solubilization methods was excellent $\left(R^{2}=0.96\right)$. Between-experiment CV's were generally less than $20 \%$, and often less than $10 \%$. Between-sample CV's were generally less than $20 \%$. CV's were consistently lower for the solubilization method. Thus, all methods were repeatable and correlated well, but the solubilization method was more precise and discriminating.
\end{abstract}

A variety of in vitro tests has previously been used to assess the cytotoxicity of dental alloys. Niemi and Hensten-Pettersen (1985) used agar overlay techniques to measure the cytotoxicity of 16 alloys and found that multiple-phase alloys showed increased toxicity. They also discovered that pre-treatment of single-phase alloys with artificial saliva reduced toxicity. Woody et al. (1977) used direct contact of alloys with previously established L929 and HeLa monolayers to evaluate $\mathrm{Ni}$-Cr and FeCr alloys; they assessed toxicity using morphological changes. They found that samples pressed from powder exhibited greater toxicity than those which were cast. Cast samples showed very little toxicity. Naji and Harmand (1990) used direct contact of a $\mathrm{Co}-\mathrm{Cr}$ alloy with human osteocytes and fibroblasts, and measured toxicity with total cell protein, alkaline phosphatase activity, and cell morphology. They determined that the surface condition of the alloy was critical to its cytotoxic behavior; microbead-blasted and -polished surfaces were more toxic than RE-sputtered or rough-cast specimens. Exbrayat et al. (1987) used attachment of human gingival cells to a Ni-CrMo alloy as a test of cytotoxicity. They measured toxicity using fibronectin and collagen production. This alloy appeared to decrease type III collagen production but not fibronectin or type I collagen production. Bumgardner et al. (1989) used gingival fibroblasts in direct contact with $\mathrm{Cu}-\mathrm{Zn}, \mathrm{Cu}-\mathrm{Al}$, and traditional gold dental alloys, and measured toxicity using trypan blue exclusion and ${ }^{3} \mathrm{H}$-thymidine uptake. Only the $\mathrm{Cu}-\mathrm{Zn}$ alloy caused altered cellular morphology, but all alloys inhibited ${ }^{3} \mathrm{H}$ thymidine uptake. They also noted a variability in the test, and observed different effects if the cells were grown in different culture media.

Craig and Hanks (1988) devised an in vitro alloy toxicity test using murine fibroblasts in direct contact with alloys. Rather than adding alloys to established cell monolayers, they allowed a suspension of cells to proliferate in the presence of the alloys. Cytotoxicity was then assessed by use of NBT (2,2'-di-p-nitrophenyl-5,5'-diphenyl-3,3'-dimethoxy-[3,3'-dimethoxy-4,4'biphenylene] ditetrazolium chloride) and di-sodium succinate for estimation of succinate dehydrogenase activity (and cell viability). A ring of inhibition of growth around the alloy, as well as densitometric and visual intensity of the stained monolayer, were used for quantification of the toxic effect. Using this procedure, they were able to show that a series of dental casting alloys could be ranked according to toxicity. In addition, they found that as-cast casting alloys were more toxic than polished alloys, but that porcelain- fused-to-metal alloys were not toxic in either the as-cast or polished condition. A subsequent study of over 30 alloys and pure metals (Craig and Hanks, 1990) further demonstrated the ability of this test to discriminate the cytotoxicity of alloys. The dissolution properties of several of these alloys in cell culture medium has also been reported (Wataha et al., 1991).

The value of any of these in vitro screening tests is dependent upon the repeatability of the test, reasonably good precision among samples for a given test, and an interpretable measure of cytotoxicity. Repeatability can be difficult to obtain because of the many potential variables which can bias the results between tests. Moreover, test variation among samples for a given test is often large enough to obscure the results without a large number of sample replications. Finally, several methods used to assess cytotoxicity may give incongruous results even within the same experiment. The purposes of this study were to attempt to validate the alloy cytotoxicity test of Craig and Hanks by making test improvements and determining the 
TABLE 1: COMPOSITIONS OF ALLOYS

\begin{tabular}{lcccccc}
\hline \multicolumn{6}{c}{ At \% Composition } \\
Alloy & $\mathrm{Ag}$ & $\mathrm{Au}$ & $\mathrm{Cu}$ & $\mathrm{Pd}$ & $\mathrm{Zn}$ & Other \\
\hline 1 & 11.5 & 49.8 & 31.5 & 2.6 & 3.2 & $\mathrm{Pt} 1.4$ \\
2 & 30.9 & 32.0 & 27.2 & 8.1 & 1.9 & \\
3 & 41.7 & 11.8 & - & 23.0 & 6.8 & $\ln 16.7$ \\
4 & 62.7 & 1.6 & 10.6 & 22.5 & 0.8 & $\ln 1.8$ \\
5 & 15.2 & 46.6 & 25.6 & - & 3.4 & $\mathrm{Cd} 9.0$ \\
6 & - & 51.5 & - & 3.8 & - & Ga12.6, In 3.9, Ni 28.1 \\
7 & 24.8 & 42.8 & 25.3 & 3.8 & 3.1 & \\
8 & 26.7 & 50.1 & - & 14.3 & 2.3 & $\operatorname{In} 5.3, \mathrm{Sn} 1.3$ \\
9 & 30.0 & 36.4 & 24.2 & 6.0 & 3.3 & \\
10 & 54.9 & - & 18.3 & 22.8 & 3.0 & B 0.9, Ru 0.001 \\
\hline
\end{tabular}

repeatability of and correlation between and among the various measures of cytotoxicity. More specifically, the aims of this study were to: (1) develop a method of solubilizing histochemically produced dyes to provide a faster, more accurate, and more precise measurement of toxicity; (2) assess the betweensample and between-experiment variability of these measures of toxicity; and (3) evaluate the correlation between and among these measures of toxicity.

\section{MATERIALS AND METHODS}

Ten alloys were used in these experiments (six samples of each alloy). The compositions of the alloys are listed in Table 1. Compositions were provided by the manufacturer and were chosen to provide a range of cytotoxicities. Common high-gold, low-gold, and Ag-Pd casting alloys as well as dental solders were included to provide clinical relevance. Fig. 1 shows the dimensions of the samples used in the cytotoxicity tests. The sample thickness was such that $0.5 \mathrm{~mL}$ of cell culture medium would come into contact with the alloy below the top of the disc portion of the sample (giving an area of exposure of $65 \mathrm{~mm}^{2}$ ). Negative controls (traditionally considered non-cytotoxic) for these tests were titanium (Ti) and Teflon (Tf). Ti samples were polished and cleaned, while Tf samples were only cleaned. These negative controls allowed the potential cytotoxicity of the polishing or cleaning procedures to be monitored. Plasticized polyvinyl chloride (PVC, with di-octyl phalate as a plasticizer) was utilized as a positive control (traditionally considered cytotoxic).

Polishing and cleaning procedures for the alloys have been described in detail previously (Watahaet al., 1991). Cells used in these experiments were Balb/c 3T3 mouse fibroblasts (ATCC CCL 163), which were anchorage-dependent, aneuploid, and contact-inhibited. Experimental cell culture medium consisted of Dulbecco's Modified Eagle Medium (DMEM) without glutamine (Sigma, St. Louis, MO), 3\% NuSerum (Collaborative Research, Bedford, MA), $28 \mathrm{mmol} / \mathrm{LHEPES}(\mathrm{pH}=7.2$ ), penicillin (125 units/mL, Gibco, Grand Island, NY), streptomycin (125 $\mu \mathrm{g} /$ $\mathrm{mL}$, Gibco), gentamycin ( $10 \mu \mathrm{g} / \mathrm{mL}$, Flow Laboratories, McLean, VA), and glutamine ( $2 \mathrm{mmol} / \mathrm{L}$, Gibco). Fibroblasts were maintained in the same medium without HEPES. Cells were harvested prior to confluence by means of a sterile trypsin/EDTA

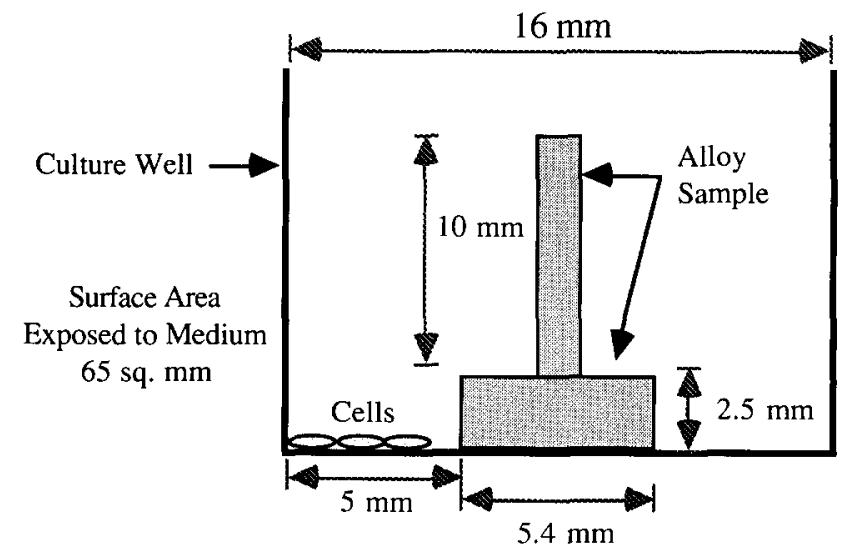

Fig. 1. Diagram of alloy sample and culture well dimensions. Culture medium covered approximately $65 \mathrm{~mm}^{2}$ of each polished alloy surface. Cells grew on the bottom of the well as close to the alloy as the alloy would allow.

solution ( $0.05 \%$ trypsin- $0.02 \%$ EDTA in normal saline), then resuspended in cell culture medium, and diluted to 100,000 cells/ $\mathrm{mL}$ with medium. One-half milliliter of the resulting cell suspension was deposited into each well of a sterile 24-well culture dish with a bottom surface area of approximately $2 \mathrm{~cm}^{2}$. This gave a cell density of $25,000 \mathrm{cell} / \mathrm{s} / \mathrm{cm}^{2}$ in each well.

Immediately after the cells were plated, the alloy samples were secured into the centers of the culture wells. Care was taken not to allow the medium to come into contact with the unpolished surfaces of the alloy samples. The culture wells were then incubated at $37^{\circ} \mathrm{C}$ for $72 \mathrm{~h}$ in a $5 \% \mathrm{CO}_{2} / 95 \%$ air atmosphere at $100 \%$ relative humidity. During this time, the cells grew on the bottoms of the culture wells as close to the alloy as the alloy would allow (Fig. 1).

After $72 \mathrm{~h}$ of incubation, phase-contrast photomicrographs were taken of cells adjacent to the alloy surfaces. The medium around each alloy was removed with a Pasteur pipette, and $1 \mathrm{~mL}$ of phosphate-buffered saline (PBS) was added to each well. The wells were swirled gently, then the PBS was replaced with 0.5 $\mathrm{mL} /$ well of MTT (3-[4,5-dimethylthiazol-2-yl]-2,5-diphenyl tetrazolium bromide)-sodium succinate solution. The composition of this solution was taken from Pearse (1972). MTT was used in place of the NBT used previously by Craig and Hanks (1988), because its formazan was easily solubilized, it produced more intense staining with shorter incubation times, and its monotetrazolium structure precluded inaccuracies from mixed conversions of the di-tetrazolium. The alloys and MTT solution were incubated at $37^{\circ} \mathrm{C}$ for $2 \mathrm{~h}$ in a $5 \% \mathrm{CO} / 95 \%$ air atmosphere. At the end of the incubation period, the MTT solution was removed and replaced with $0.5 \mathrm{~mL} /$ well of $4 \%$ formalin in $0.2 \mathrm{M}$ Tris buffer at $\mathrm{pH} 7.2\left(\right.$ at $\left.25^{\circ} \mathrm{C}\right)$ for $10 \mathrm{~min}$. The wells were then washed twice with double-distilled water and dried with a hair dryer for 10 min.

With the alloys still in the culture wells, photomicrographs were taken of the MTT-stained cells at the cell-alloy interface, and the rings of inhibition were measured. The alloys were then removed, and the wells were scanned with a densitometer for assessment of the percent transmittance of light through the stained areas and the central areas (formerly occupied by the alloy samples). Photographs were taken of the wells to preserve a visual record of their gross appearance, then $1.0 \mathrm{~mL} / \mathrm{well}$ of a $6.25 \% \mathrm{v} / \mathrm{v}$ $0.1 \mathrm{~mol} / \mathrm{L} \mathrm{NaOH}$ in DMSO solution was added to each well. The wells were swirled for 5 min until the purple color was uniform, the contents were aspirated directly into a Beckmann DU-64 spectrophotometer, and the absorbance was evaluated at $560 \mathrm{~nm}$. 


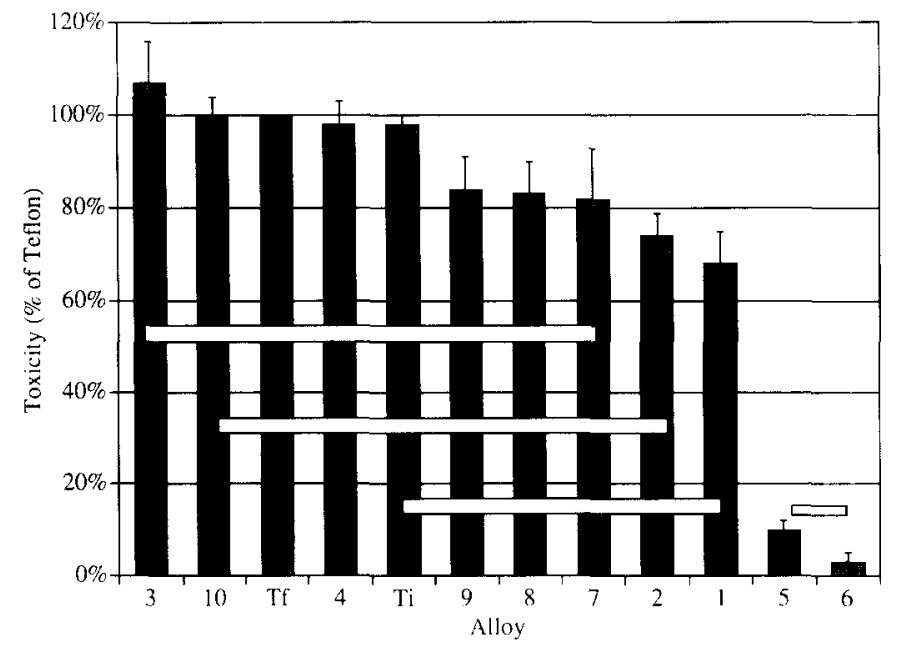

Fig. 2. Cytotoxicity of alloys as measured by densitometer. Toxicity is expressed as a percentage of the Teflon (Tf) controls. Error bars are one standard error of the mean $(n=4)$. Horizontal connecting bars signify Tukey groups $(p=0.05)$. There was poor separation of statistical groups when this method was used.

The graphical densitometer output was converted to numerical data by calculation of the difference in percent transmittance between the center area (where the alloy sample was) and the periphery (where the cells were). The transmittance on both sides of the center area was estimated and averaged. The transmittance in the central area was then subtracted from this average, and the absolute value of the difference was taken. These values were averaged for the six samples of each type of alloy, and this average was expressed as a percentage of the Tf negative control.

The staining density was rated visually by use of the gross culture plate photographs according to criteria shown in Table 2 . All six samples of a given type of alloy were evaluated simultaneously for assignment of a single visual rank value.

The six absorbance values obtained from the DMSO-solubilized formazan for each alloy were averaged, and these averages were expressed as a percentage of the Tf negative control.

Two types of variation were calculated for each alloy type. First, the between-sample variation for a particular toxicity parameter was calculated by averaging the coefficients of variation generated for each alloy type in each experiment. Second, between-experiment variation was obtained by averaging alloy toxicity over all the experiments, then computing the coefficient of variation. Differences in the alloy toxicities were assessed by one-way ANOVA and Tukey multiple-comparison intervals $(\mathrm{p}=$ 0.05 ). A correlation between the results for densitometer and spectrophotometer data was assessed by simple linear regres-

TABLE 2: CRITERIA FOR VISUAL CYTOTOXICITY RANKING

Rank Description

$1 \quad$ Even staining across wells similar to blank; staining intensity similar to blank.

2 Generally even staining in most wells; some areas of less intense stain or slight unevenness of stain in some wells.

3 Uneven staining in most wells and/or less intense staining in most wells.

$4 \quad$ Extremely uneven staining in most wells; little staining intensity.

$5 \quad$ Little or no evidence of stain in any well.

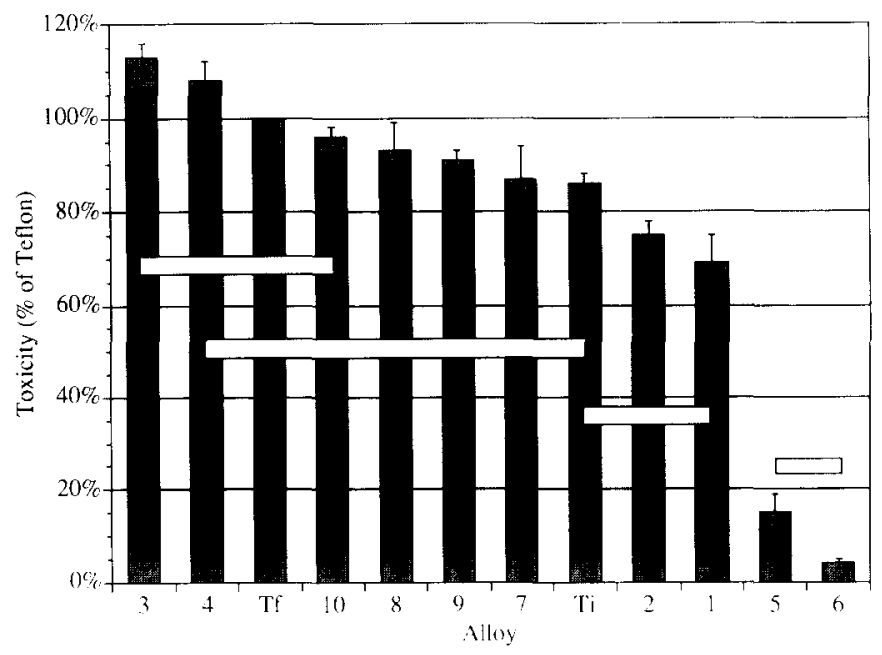

Fig. 3. Cytotoxicity of alloys measured spectropholometrically. Layout for this graph is identical to Fig. 2. Note the more definitive statistical separation of groups when compared with Fig. 1.

sion. Comparison of visual data with the densitometer and spectrophotometer data was done subjectively.

\section{RESULTS}

Results for the densitometer measurements are shown in Fig. 2. The alloys were sorted from best to worst. The results for the PVC positive controls (not shown) consistently showed zero cell staining. The densitometer separated the alloys into five groups, although these groups were often not distinguishable statistically. Alloy 3 appeared to be slightly but not significantly better than Tf; alloys 4, 10, and Ti appeared to be equivalent to Tf; and alloys 9,8 , and 7 appeared to be slightly but not significantly worse than Tf. Alloys 2 and 1 were significantly worse than Tf, while alloys 5 and 6 showed the greatest cytotoxicity and were much worse than the Tf controls. The Tukey multiple-comparison tests were unable to separate these groups completely because of the relatively large variation with this method of evaluation.

Results for the spectrophotometer measurements are shown in Fig. 3. The same five basic groups were delineated by the spectrophotometer as with the densitometer. However, the statistical identity of these groups was improved when the

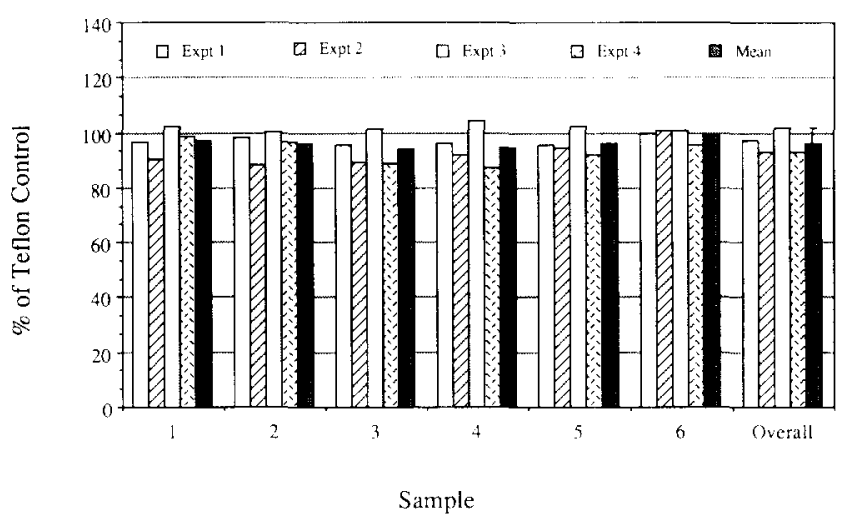

Fig. 4. Between-sample variation for alloy 10. Each of the first six columnar groups represents data from one alloy sample for four experimental repetitions. The seventh columnar group shows the means of the six samples for each experiment. The dark bar in each group is the mean of the other four bars. The error bar associated with the overall mean represents one standard error of the mean. Between-sample variation was low. Variation of this magnitude was typical of most alloys studied. 


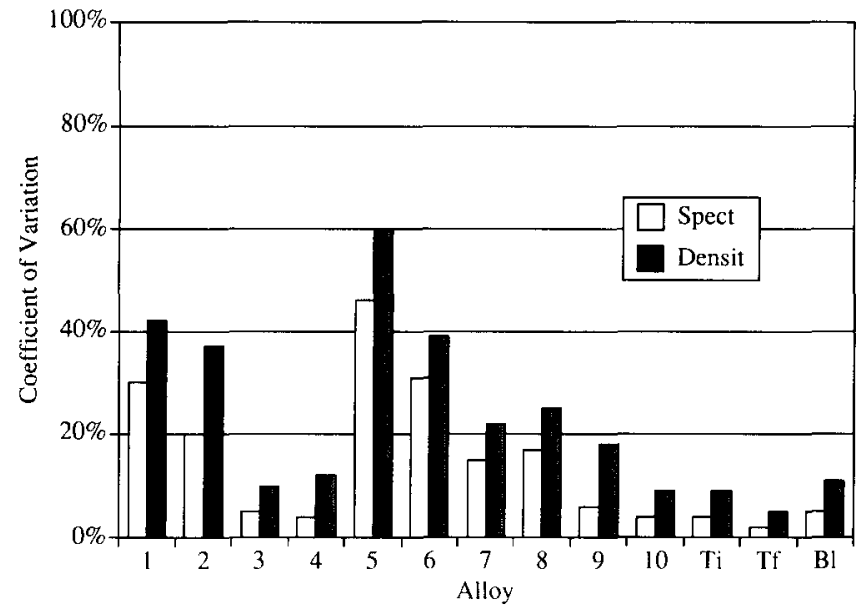

Fig. 5. Between-sample variation, all alloys. The coefficients of variation (CV's) represent an average of four experiments. The large CV's observed for alloys five and six are mathematical artifacts caused by the extreme toxicity of these alloys. Variations with densitometric readings were generally greater than those taken spectrophotometrically. Overall, between-sample variation was acceptable; alloys 1 and $\mathbf{2}$ were worse than the others.

spectrophotometer was used. Alloys 3 and 4 were better but not significantly better than Tf, while alloy 10 appeared to be equivalent to Tf. Alloys $9,8,7$, and Ti were slightly but not significantly worse than the Tf; alloys 2 and 1 were significantly worse than Tf; and alloys 5 and 6 showed the most severe cytotoxicity. A comparison of Figs. 2 and 3 shows that the Tukey groupings were cleaner for the spectrophotometric data, which was largely a result of smaller between-experiment variation when this method was used. Ranking of the alloys was similar in the two tests, but there were some differences: Alloy 4 appeared to be superior to $\operatorname{Tf}$ (by spectrophotometric measurements), while $\mathrm{Ti}$ appeared to be slightly worse.

The results from the visual assessments are shown in Table 3. Here, four groups were apparent. Alloys 10 and Ti were indistinguishable from the Tf controls (ratings of 1.0). Alloys $9,4,3,8$, and 7 were slightly worse than the Tf(ratings of 1.5-2.0), alloys 1 and 2 were markedly worse than $\mathrm{Tf}$ (ratings of 3.0 ), and alloys 5 and 6

TABLE 3: CYTOTOXICITY OF ALLOYS MEASURED VISUALLY

\begin{tabular}{cccccc}
\hline \multicolumn{5}{c}{ Experiment } \\
Alloy & 1 & 2 & 3 & 4 & Average \\
\hline $\mathrm{Tf}$ & 1 & 1 & 1 & 1 & 1 \\
$\mathrm{Ti}$ & 1 & 1 & 1 & 1 & 1 \\
10 & 1 & 1 & 1 & 1 & 1 \\
9 & 2 & 2 & 1 & 1 & 1.5 \\
4 & 2 & 1 & 1 & 2 & 1.5 \\
3 & 2 & 2 & 1 & 2 & 1.75 \\
7 & 3 & 2 & 2 & 1 & 2 \\
8 & 3 & 1 & 2 & 2 & 2 \\
1 & 3 & 3 & 3 & 3 & 3 \\
2 & 3 & 3 & 3 & 3 & 3 \\
5 & 4 & 4 & 4 & 4 & 4 \\
6 & 5 & 5 & 5 & 5 & 5 \\
\hline
\end{tabular}

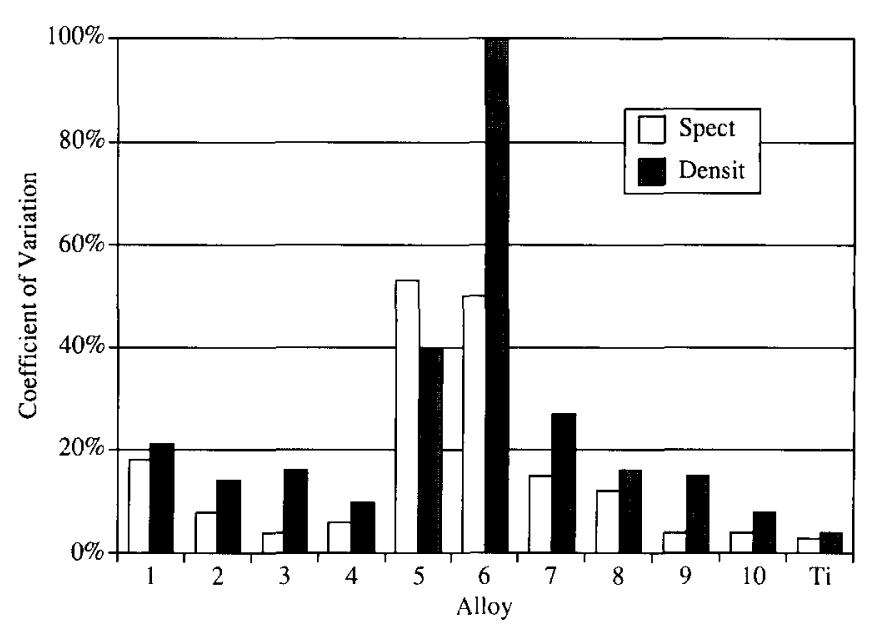

Fig. 6. Between-experiment variation, all alloys. Coefficients of variation (CV's) represent an average of four experiments. The large CV's observed for alloys five and six are mathematical artifacts caused by the extreme toxicity of these alloys. Variation with densitometric readings were generally greater than those taken spectrophotometrically. Overall, between-experiment variation was acceptable.

were much worse than the Tf controls (ratings of 4-5). No group exhibiting better staining than Tf was discernible. However, the overall ranking of the alloys by this method corresponded reasonably well to the densitometer and spectrophotometer rankings (Figs. 2 and 3 ).

The method of evaluating alloy cytotoxicity with "ring of inhibition" measurements was abandoned after initial experimentation. The method seemed particularly susceptible to systematic errors, gave paradoxical results, and was time-consuming to perform.

Between-sample variation in these experiments was generally low. Fig. 4 shows the between-sample variation for alloy 10 , which was typical of most alloys. In this Fig., the first six groups of columns represent the toxicity as measured by formazan absorbance for each of the six samples in the four experimental replications. The seventh columnar group shows the average toxicity for all six samples for each experiment. In addition, the darkly shaded bar in each columnar group shows the mean of the other four bars. The overall mean in the seventh column has an error bar (1SEM) reflecting the overall precision for the experiment. Using this overall mean and its error bar, one can compare the behavior of each sample with the overall behavior of the alloy, either experiment by experiment or as an average of all four experiments. It is apparent from Fig. 4 that no sample showed behavior which was significantly different from the overall mean of the other samples. Eight of the ten alloys tested showed results essentially similar to that of alloy 10 . Alloys 1 and 2 showed more deviant behavior, but variation was not so great as to compromise the results.

Fig. 5 shows between-sample coefficients of variation for all alloys. Both densitometer and spectrophotometer evaluations are shown. It is apparent from this Fig. that densitometer and spectrophotometer measurements gave similar magnitudes of between-sample variation for each alloy. However, betweensample variations were consistently smaller when measured by the spectrophotometer. The between-sample variation appeared to be larger for some alloys $(1,2,5$, and 6$)$.

Fig. 6 shows between-experiment coefficients of variation for all alloys. In general, the repeatability of the experiment appeared to be very good. Alloys 5 and 6 had significantly higher degrees of variation, due to the fact that the minimal staining approached the limits of detection of both the spectrophotometer 


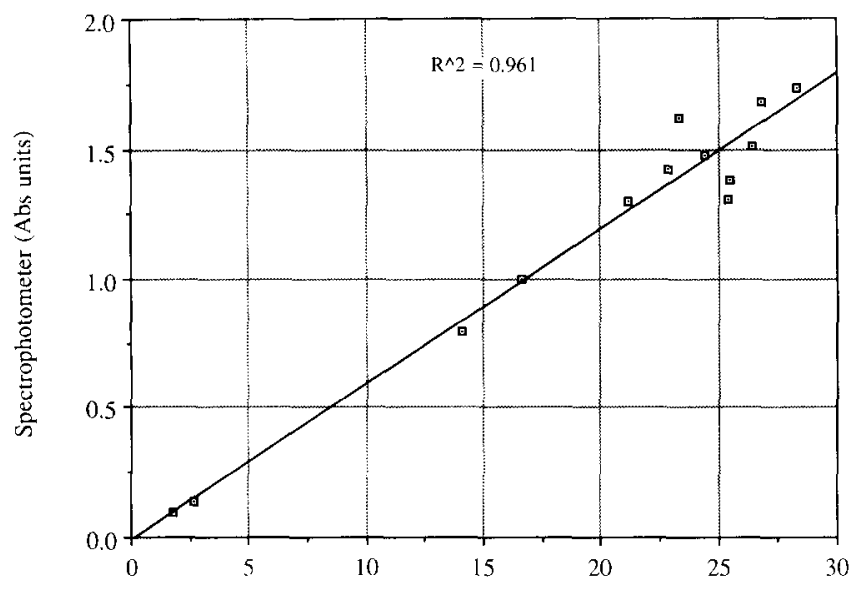

Densitometer

Fig. 7. Statistical comparison of densitometric and spectrophotometric methods of toxicity evaluation. These two methods showed excellent statistical agreement $\left(\mathbf{R}^{2}=\right.$ 0.96). Aberrations were probably due to difficulties in interpretation of graphical output of the densitometer.

and densitometer. Thus, the means approached the magnitudes of the standard deviations for these alloys, resulting in large coefficients of variation. The actual standard deviations for these alloys were of a magnitude similar to those of the other alloys. Other alloys generally showed variation of less than $20 \%$, and many showed variations of $10 \%$ or less. It was again apparent that the spectrophotometer showed consistently less variation than the densitometer for all alloys except alloy 5 .

The spectrophotometric and densitometric methods were compared by simple linear regression; these results are shown in Fig. 7. This regression represents data from a single alloy toxicity experiment, but is representative of all four experiments. The correlation between densitometer and spectrophotometer measurements was very good $\left(R^{2}=0.96\right)$.

\section{DISCUSSION}

Two types of variation were assessed in the alloy toxicity experiments: between-sample variation and between-experiment variation. A small between-sample variation is important to ensure consistent results between alloy samples of the same type and obviates the need for using large numbers of samples to detect differences between alloy types. Between-sample variation might arise from (1) differences in composition or polishing between alloy samples, or (2) culture artifacts, such as chemical or biological contamination, aspiration or washing damage, or uneven cell-plating densities. The contribution of these artifacts as sources of variation was assessed by use of negative controls or blank wells. Fig. 5 shows that the controls (Ti and Tf), blanks, and several of the alloys had overall coefficients of variation of less than 5\% (with spectrophotometric measurements); thus, confidence in the repeatability of the cell culture and processing procedures was assumed.

Alloys 5 and 6 had large between-sample variations (Fig. 5). This was due to the very low cell growth around these alloys, such that the mean cell growth was of a magnitude similar to the standard deviations. As described earlier, the between-sample variation of alloys 5 and 6 was really no worse than that for the other alloys. Alloys 1,2, 7, and 8 showed higher between-sample variation, in spite of cell growth well above the detection limits. If one assumed that this was not due to cell culture or processing artifacts (such as plating, aspiration, or washing variation), then the variation must have come from a difference between samples.
Such an assumption would seem to be tenable in light of the blank and control results (which showed very little betweensample variation). Alloys 2, 7 , and 8 showed no pattern to the between-sample variation which was observed; this suggested an occasional and random chemical or biological contamination of the samples. Alloy 1 did exhibit a between-sample variation pattern, in that two of the samples showed consistently higher toxicities. Such patterned variation tended to indicate compositional or polishing differences between samples, although chemical and biological contamination could have been superimposed. An additional possibility is that the release of metal cations from alloys $1,2,7$, and 8 was variable, depending upon polishing from sample to sample. Although every attempt was made to standardize the polishing procedure, this is still a possibility.

Overall, between-sample variation was sufficiently small to warrant confidence in the alloy toxicity test results. Even in cases where the variation was larger, it was not so large as to limit the ability of the test to rank the alloy reliably among other alloys. Six samples appeared sufficient to facilitate such ranking; fewer would have been needed in cases where the betweensample variation was less than $5 \%$ (alloys $3,4,9$, and 10 ).

The second type of variation was between-experiment variation. In any in vitro screening test, experimental repeatability is paramount. Such variation between experiments might arise from such factors as cell line passage number or cell activity, changes in the measuring instrumentation (densitometer, spectrophotometer), or differences in the cleaning procedure. The cleaning procedure would be more likely to cause betweenexperiment variation than would the polishing procedures, because the alloys were cleaned in batch fashion, but were polished individually. Polishing differences would thus tend to manifest themselves as between-sample variation. Since the cell densities or absorbances were normalized to the Tf controls, factors such as MTT incubation times, fixing times, and differences in cell growth rates probably had little effect on the between-experiment variation.

As shown in Fig. 6, experimental repeatability appeared to be very good for the majority of the alloys. If one considers the potential number of causes of such variation in cell monolayer experiments, variations of this magnitude were quite acceptable, particularly with the spectrophotometer measurements. Two notable exceptions to this statement were alloys 5 and 6 . The cause of these higher coefficients of variation were discussed earlier.

Figs. 2 and 3 and Table 3 show that there was generally good agreement between the densitometric, spectrophotometric, and visual methods of evaluation, although the statistical groupings varied slightly by each method of evaluation. Fig. 7 shows a strong positive correlation between these tests, indicating that solubilization of the MTT-formazan dye was an acceptable alternate method of cytotoxicity quantification. Deviations from an ideal correlation were probably caused by misrepresentations of cell density inherent in the densitometric graphic analysis. The "ring of inhibition" measurements were not generally repeatable and did not correlate with the other three methods. The general agreement among the first three methods tended to validate them as reliable methods of cytotoxicity assessment from a statistical perspective.

Each method of evaluation appeared to have its advantages and disadvantages. The densitometric method had the advantage that the stained monolayer was retained for future reference if needed. However, this method had several disadvantages. First, it assumed that the scan path across the plate was representative of the entire stained area. If non-uniform growth 
occurred, this assumption was violated. The method also required that the sample be precisely centered in the well, so that the scan path would correctly register the clear area where the sample had been. An off-center sample caused the scan path to include a portion of the stained monolayer, giving an artificially high density to the sample area. Second, the computation of monolayer density required subjective evaluation of the graphical output from the plotter. Although this was trivial in the ideal cases, it was difficult in cases where a toxic response had caused less uniform cell growth.

There were several advantages with the spectrophotometric method. First, the solubilization of the dye from the entire well gave a more accurate representation of total cell activity than densitometric measurements, which scanned only a fraction of the well. Second, it provided a more objective assessment of cell density, since the method did not involve graphical interpretation. These two advantages produced less-variable data, which led to better discrimination between alloys. Third, the spectrophotometric method did not require that the sample be precisely centered, since the entire well was assessed and no scan path was involved. Finally, this method was faster than the densitometric method.

The spectrophotometric method did have several disadvantages. First, because the dye was solubilized, no record of the staining pattern remained in the wells after solubilization. This limitation was largely mitigated by photographs of the wells, which adequately preserved these data for future reference. Second, this method required that all samples be the same diameter, since the quantity of dye produced was proportional to the cell growth area. Densitometric measurements, which assessed cell staining density, did not have such a requirement. In practice, this limitation was not restrictive, since samples were easily constructed with the same diameters. Furthermore, a variation of $0.5 \mathrm{~mm}$ in sample diameter resulted in only a $2 \%$ change in cell growth area. Thus, small variations in diameter were generally acceptable within the framework of the variation of this test.

The advantages of the visual method were simplicity and speed. Results with a trained observer could be obtained in several minutes without expensive equipment. There were disadvantages with this method, however. First, the data obtained were essentially non-parametric, since the categories created to rate the cell density were arbitrary. This fact precluded parametric statistical comparisons with the other methods, and did not allow for assessment of the between-sample and between-experiment variations. Second, the method lacked the discrimination of the other two methods. This was also a consequence of the non-parametric nature of the data. Finally, the results were dependent upon the observer's interpretation. In the absence of an attempt to standardize or adjust for different observer ratings, comparisons of data obtained from different observers would be difficult.

In summary, (1) The use of MTT rather than NBT as a histochemical stain allowed stained monolayers to be solubilized and then evaluated by use of a spectrophotometer. MTT required less incubation time for staining, gave more intense stains, was less expensive, and did not have the complications of a partially reduced di-tetrazolium molecule.

(2) Densitometer scanning, visual assessment, and spectrophotometric evaluation ranked the in vitro toxicities of a wide variety of alloy compositions similarly. However, "ring of inhibition" measurements were not as reproducible and did not correlate well with the other three methods. Statistical correlation between the densitometric and spectrophotometric methods was excellent $\left(R^{2}=0.96\right)$.

(3) Between-sample variation was generally less than $20 \%$; spectrophotometric variation was consistently lower than for the densitometric method. Adequate test discrimination was realized with six replicate samples; fewer would have been needed with alloys which had between-sample coefficients of variation of less than $10 \%$.

(4) Between-experiment variation coefficients of variation were generally less than $20 \%$ and often less than $10 \%$. This established confidence in the accuracy of any given repetition of the experiment.

(5) Each method of cytotoxicity measurement had its strengths and weaknesses. The visual method gave accurate results with a minimum amount of equipment, but required some observer training and provided only non-parametric data. Densitometric evaluations were easy to obtain and preserved the monolayer for future reference. However, this method was timeconsuming and less precise. Spectrophotometric evaluation of the solubilized formazan dye was fast, objective, and had the least variation. It required more expensive equipment and destroyed the stained monolayer, however.

\section{ACKNOWLEDGMENT}

This investigation was supported by USPHS Grants 5 T32 DE07057, 1 P50 DE09296, and RO1 DE07987 from the National Institute of Dental Research, Bethesda, MD 20892.

Received April 1, 1991/Accepted July 5, 1991

Address correspondence and reprint requests to:

J.C. Wataha

The University of Michigan

School of Dentistry BMS Dept

1011 N. University Ave

Ann Arbor, MI 48109-1078

\section{REFERENCES}

Bumgardner JD, Lucas LC, Tilden AB (1989). Toxicity of copperbased dental alloys in cell culture. $J$ Biomed Mater Res 23:1102-1114.

Craig RG, Hanks CT (1988). Reaction of fibroblasts to various dental casting alloys. J Oral Pathol 17:341-347.

Craig RG, Hanks CT (1990). Cytotoxicity of experimental casting alloys evaluated by cell culture tests. J Dent Res 69:1539-1542.

Exbrayat P, Couble ML, Magloire H, Hartman DJ(1987). Evaluation of the biocompatibility of a Ni-Cr-Mo dental alloy with human gingival explant culture in vitro: morphological study, immunodetection of fibronectin, and collagen synthesis. Biomaterials 8:385-392.

Naji A, Harmand MF (1990). Study of the effect of the surface state on the cytocompatibility of a $\mathrm{Co}-\mathrm{Cr}$ alloy using human osteoblasts and fibroblasts. J Biomed Mater Res 24:861-871.

Niemi L, Hensten-Pettersen A (1985). In vitro cytotoxicity of AgPd-Cu based casting alloys. J Biomed Mater Res 19:549-561.

Pearse AG (1972). Histochemistry: theoretical and applied. Volume 2. Baltimore: Williams and Wilkins, Chapter 20.

Wataha JC, Craig RG, Hanks CT (1991). The release of elements of dental casting alloys into cell culture medium. J Dent Res (70:1014-1018).

Woody RD, Huget EF, Horton JE (1977). Apparent cytotoxicity of base metal casting alloys. J Dent Res 56:739-743. 